\title{
Economía circular, una estrategia para el desarrollo sostenible. Avances en Ecuador
}

Circular Economy, a Strategy

for Sustainable Development. Advances in Ecuador

Economia circular, uma estratégia

para o desenvolvimento sustentável. Avanços no Equador

\author{
Marcia Almeida-Guzmán \\ Universidad Andina Simón Bolívar (UASB). Quito, Ecuador \\ marcia.almeida@uasb.edu.ec \\ ORCID: https://orcid.org/0000-0001-6123-6971
}

César Díaz-Guevara

Universidad Andina Simón Bolívar (UASB). Quito, Ecuador

cesardiazguevara@gmail.com

ORCID: https://orcid.org/0000-0003-0945-4634

DOI: https://doi.org/10.32719/25506641.2020.8.10

Recibido: 8 de abril de 2020 - Revisado: 11 de abril de 2020

Aceptado: 28 de mayo de 2020

Artículo de investigación

Licencia Creative Commons

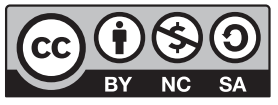




\section{Resumen}

Hoy más que nunca urge un sistema económico más amigable con el ambiente, menos depredador y consciente del bienestar de los habitantes de este planeta, por ello el propósito de este artículo es explorar en la literatura la perspectiva conceptual de la economía circular, su aporte estratégico al desarrollo sostenible, su implantación y consecuencias, así como también las opiniones contrarias. Tema de progresivo interés y tendencia mundial, que ha generado un notable movimiento, a nivel de gobiernos, fundaciones, industria y sociedad, respecto a reconsiderar la gestión de los recursos a través de todo el ciclo de vida de un producto/servicio que conlleve a lograr prosperidad, atenuando el impacto en el medioambiente y posibilitando el bienestar de los seres humanos y demás especies. La metodología de investigación es de tipo cualitativa, y a través de un método exploratorio descriptivo se reseñan los hallazgos. Se concluye que en las estrategias de desarrollo sostenible, tanto el gobierno como las organizaciones ecuatorianas deberían considerar la implementación de proyectos de economía circular y definir acciones a tomar desde la esfera pública para fomentar la transición hacia esta nueva concepción económica. Una limitación de este estudio es la escasa evidencia empírica local sobre el tema, por lo que se ha recurrido a literatura y experiencias internacionales.

Palabras clave: Economía circular, gestión de recursos, ciclo de vida, producto/servicio, estrategia de diseño sostenible.

JEL: Q01 Desarrollo sostenible.

\section{Summary}

Today more than ever a more environmentally friendly economic system is urgent, less predatory and aware of the well-being of all people in the planet. Therefore, the purpose of this article is to explore in literature the conceptual perspective of circular economy, its strategic contribution to sustainable development, its implementation and consequences, as well as contrary opinions. A progressive interest and global trend topic, which has generated a notable movement at the level of governments, foundations, industry and society, regarding reconsidering resources management throughout the life cycle of a product/ service that leads to achieve prosperity, mitigating the impact on the environment and enabling the well-being of human beings and other species. The research methodology is qualitative, and the findings are reviewed through a descriptive exploratory method. Concluding that in the strategies of sustainable development, both the government and Ecuadorian organizations should consider the implementation of circular economy projects and define actions to be taken from the public sphere to promote the transition towards this new economic conception. A limitation of this study is the scarce local empirical evidence on the subject, thus international literature and experiences have been used.

Keywords: Circular economy, resource management, life cycle, product / service, sustainable design strategy.

JEL: Q01 Sustainable development. 


\section{Resumo}

Hoje mais do que nunca se faz necessário um sistema econômico mais amigável ao meio ambiente, menos depredador e mais consciente em relação ao bem-estar dos habitantes do planeta. Por conta disso, o objetivo deste artigo é explorar, na literatura, a perspectiva conceitual da economia circular, sua contribuição estratégica para o desenvolvimento sustentável, sua implantação e suas consequências, assim como as opiniões a ela contrárias. Trata-se de um tema de crescente interesse e já uma tendência mundial, que tem gerado uma notável movimentação no que corresponde a governos, fundações, indústria e sociedade em se tratando de reconsiderar a gestão dos recursos através de todo o ciclo de vida de um produto/serviço de forma a se obter prosperidade e atenuar o impacto sobre o meio ambiente, possibilitando o bem-estar dos seres humanos e das demais espécies. A metodologia de pesquisa é do tipo qualitativa, e os resultados são apresentados através de um método exploratório descritivo. Conclui-se que as estratégias de desenvolvimento sustentável tanto do governo quanto de organizações equatorianas deveriam considerar a implementação de projetos de economia circular e definir ações a serem tomadas a partir da esfera pública com o fim de fomentar a transição para essa nova concepção econômica. Uma limitação deste estudo é a escassa evidência empírica local sobre o tema. Em vista disso, a literatura sobre o tópico e experiências internacionais foram utilizadas.

Palavras-chave: Economia circular, gestão de recursos, ciclo de vida, produto/serviço, estratégia de design sustentável.

JEL: Q01 Desenvolvimento sustentável.

\section{Introducción y estado de la cuestión}

E 1 tradicional sistema de producción basado en la extracción de los recursos, el procesamiento no amigable de materias primas para convertirlas en productos a nivel de las empresas, la utilización por parte de los consumidores y la eliminación de residuos de manera inadecuada y en la mayoría de los casos contaminantes, evidentemente no es sostenible (Priede Bergamini e Hilliard 2019); por ello, desde finales de los ochenta autores como Pearce y Turner (1989) empezaron a hablar de la necesidad de un nuevo paradigma basado en una economía circular.

Paradigma que ha ido adquiriendo cada vez más importancia en diferentes ámbitos, como el académico, económico, político y social, surgiendo organizaciones como la Ellen MacArthur Foundation (2019 y 2015), que desde 2010 ha puesto a la economía circular en la pauta de los responsables de las decisiones en todo el mundo como una forma potencial para que nues- 
tra sociedad aumente la prosperidad, al tiempo que reduce la dependencia de las materias primas y la energía.

Este modelo económico, social y ambiental conlleva un nuevo reto para todos los países y organizaciones en el mundo, dado que implica el establecimiento de "un sistema económico, que busca la preservación de los recursos naturales y que tiene como propósito contribuir simultáneamente a disminuir el impacto ambiental del desarrollo, aumentar la eficiencia del uso de recursos y mejorar el bienestar de todas las partes interesadas" (INEN 2020, 7).

Se hace necesario que gobierno, productores y consumidores adopten este nuevo paradigma como una forma de generar productos desde el origen, es decir, desde su diseño hasta su logística inversa, ${ }^{1}$ lo que permitirá realizar negocios considerando el crecimiento económico de la sociedad, la sostenibilidad ambiental y la reducción de riesgos por la volatilidad e incertidumbre de precios de las materias primas y recursos energéticos (Lett 2014).

En este entorno, el modelo de economía lineal basado en extraer, producir y consumir (ES MAPAMA y MIMEINCO 2018) resulta totalmente incompatible en un mundo de recursos limitados, por cuanto el aumento perpetuo de la intensidad material y energética, así como el crecimiento del consumo no es sostenible (Fundación COTEC 2017; Varela 2018) o, como lo señalan Song Guohui y Li Yunfeng $(2012,786)$, “enorme producción, enorme consumo y enorme desperdicio".

Así, la economía circular está abriendo formas de conciliar las perspectivas de crecimiento y participación económica con las de la prudencia y equidad ambiental. Y a nivel mundial está inspirando a altos ejecutivos, empresarios, políticos, ingenieros, diseñadores (World Economic Forum 2014) y a organismos de normalización a liderar este sistema económico.

En este contexto, el objetivo de esta investigación es explorar en la literatura la perspectiva conceptual de la economía circular, su aporte estratégico al desarrollo sostenible, su implantación y consecuencias, así como el camino recorrido para conseguirla.

1. "La logística inversa está referida a la devolución de productos, el reciclaje, la sustitución de materiales, reutilización de materiales, eliminación de residuos, así como la renovación, reparación y refabricación" (Contreras, Tordecilla y Silva 2013, 154). 
Por tanto, ya viene siendo hora de establecer un sistema económico de intercambio y producción que, en todas las etapas del ciclo de vida de los productos (bienes y servicios), busque aumentar la eficacia en la utilización de los recursos, y al mismo tiempo disminuya el impacto en el medio ambiente, y aumente el bienestar de los individuos, además de que el valor de los productos, materiales y recursos se conserven en la economía el mayor tiempo posible y la generación de desechos se reduzca al mínimo. Este sistema de carácter transversal y gradual viene a ser la economía circular (Santamaría 2019).

Este modelo económico, a más de la conversión de los residuos en nuevos recursos, propone además un cambio innovador en el actual sistema de producción, respecto a que el diseño de cada fase del proceso se guíe por la idea de la regeneración (Chaves y Monzón 2018).

Se debe considerar, además, que la economía circular sugiere un modelo económico regulado según las leyes de la naturaleza como redes de componentes que interactúan, intercambio de flujos de materiales y energía, patrones de reciclaje y mimetismo ambiental (Ghisellini, Cialani y Ulgiati 2015).

\section{Marco teórico de la economía circular}

La teoría de sistemas ha permitido adaptar sistemas circulares a prácticamente todas las ciencias, entre ellas la económica, lo cual permite sostener un modelo basado en sí mismo y prescindir de las tan temidas y otrora olvidadas externalidades negativas (Varela 2018) para el medioambiente, el clima y la salud humana.

Por ello, la economía circular, concebida a partir de un ciclo de desarrollo y transformación, que evoluciona optimizando el uso de los recursos y promoviendo la eficiencia de los sistemas productivos, coadyuva a eliminar las externalidades negativas de la actividad económica, al mismo tiempo que garantiza el crecimiento económico, un mayor bienestar de la sociedad y la preservación y mejora del capital natural (ES MAPAMA y MIMEINCO 2018). 
Si bien en la literatura revisada se han planteado diversas definiciones sobre economia circular, en este artículo se consideraran aquellas más consensuadas a nivel mundial.

Así, la norma francesa AFNOR XP X30-901 (2018, 5), la cual ha sido adoptada como norma ecuatoriana, la define como el "sistema económico de intercambio y producción que, en todas las etapas del ciclo de vida de los productos/servicios, busca aumentar la eficacia de la utilización de los recursos, disminuir el impacto en el medioambiente permitiendo el bienestar de individuos, en el cual el valor de los productos, materiales y recursos se mantiene en la economía el mayor tiempo posible y la producción de desechos se reduce al mínimo".

Mientras que la Comisión Europea, en su Plan de Acción para la Economía Circular $(2015,2)$ señala: "en este modelo económico, el valor de los productos, los materiales y los recursos se mantienen en la economía durante el mayor tiempo posible, y se reduce al mínimo la generación de residuos, lo cual contribuye a lograr una economía sostenible, eficiente en el uso de los recursos y competitiva".

Por su parte Fundación COTEC (2017) considera que la adopción de una economía circular conlleva un cambio hacia sistemas regenerativos a partir de su diseño, para mantener el valor de los recursos y de los productos el mayor tiempo posible, lo que limita el uso de materias primas y energía, evitando la creación de residuos e impactos negativos derivados.

Se puede mencionar, entonces, que la economía circular se apoya en principios como la diversidad, la resiliencia y el pensamiento sistémico, ${ }^{2}$ que requieren un enfoque metabólico, integrando ciclos materiales biológicos y tecnológicos ${ }^{3}$ (Varela 2018).

2. "Capaz de describir la estructura y el comportamiento de los sistemas, identificando sus propiedades y relaciones" (Martínez y Londoño 2012, 46-47).

3. "El modelo de economía circular hace una distinción entre ciclos técnicos y biológicos. El consumo ocurre solamente en los ciclos biológicos, donde alimentos y otros materiales de base biológica (por ejemplo, algodón y madera) son diseñados para regresar al sistema mediante procesos de compostaje y digestión anaerobia. Los ciclos regeneran sistemas vivos, como el suelo, que ofrecen recursos renovables para la economía. Mientras que los ciclos técnicos recuperan y restauran productos componentes y materiales mediante estrategias de reutilización, reparación, remanufactura o (en última instancia) reciclaje" (Ellen MacArthur Foundation 2020, párr. 5). 
Para Ellen MacArthur Foundation, organización que ha destinado recursos al estudio y la implantación en Europa de la economía circular, esta "es una economía restaurativa y regenerativa por intención y por diseño para mantener productos, componentes y materiales en su máximo grado de utilidad y valor en todo momento, distinguiendo ciclos técnicos y biológicos" $(2015,46)$.

Por consiguiente, se trata de un sistema de producción y consumo que supera al sistema lineal. Y en el cual el término "caducidad" es sustituido por "restauración"; se elimina en la medida de lo posible la utilización de químicos y tóxicos que dañan la biosfera y perjudican la reutilización mediante la eliminación de residuos y la apuesta por energías renovables; todo ello mediante la optimización del diseño de productos y materiales por un lado y sistemas y modelos de negocio por otro. (Ellen MacArthur Foundation 2014, 3)

También algunos investigadores han ido definiendo a la economía circular. Pearce y Turner, en 1990, formularon literalmente el término, proponiendo un flujo económico cerrado que explicaba cómo sería su posible funcionamiento (Prieto-Sandoval, Jaca y Ormazabal 2017).

Sin embargo, de no ser un concepto nuevo, en los últimos años se ha incrementado la preocupación por el agotamiento de recursos naturales debido al sistema económico actual de "producir, consumir, desechar"; por ello, "las partes interesadas ${ }^{4}$ coinciden en la necesidad de acelerar la transición hacia un sistema económico circular más eficiente en cuanto a la utilización de los recursos que permita reducir el impacto ambiental del desarrollo y que mejore el bienestar de los individuos" (INEN 2020, iv).

Por consiguiente, "la economía circular es un ciclo de desarrollo positivo y continuo que protege y mejora el capital natural, optimiza el rendimiento de los recursos, y minimiza los riesgos del sistema al gestionar con rigor las reservas finitas y los flujos renovables" (Espaliat 2017, 27), funcionando de forma eficaz en todas las escalas, e intentando desvincular el desarrollo económico global del consumo de recursos finitos.

En la misma línea, los investigadores chinos Guohui y Yunfeng (2012) indican que la economía circular se centra en la utilización y reutilización de

4. "Persona u organización que puede afectar, verse afectada o percibirse como afectada por una decisión o actividad" (INEN 2020, iv). 
recursos, que se basa en el principio de disminución, reciclaje y extensión de los mismos. Se caracteriza, además, por un bajo consumo, baja descarga y alta eficiencia, con el consecuente uso sostenible de los recursos naturales, generando un máximo beneficio económico, social y ambiental.

Una fortaleza adicional de esta teoria económica es que no ha sido relegada por el pensamiento ortodoxo imperante (Varela 2018), en el cual a pesar de los cambios experimentados en los últimos 150 años dentro del sistema económico (como el paso del capitalismo industrial al financiero), ha perdurado un modelo de producción y consumo lineal, en el que cualquier tipo de producto/servicio es generado a partir de unas determinadas materias primas e insumos, para luego ser comercializado, utilizado y posteriormente desechado (Ellen MacArthur Foundation 2014), siendo su principal falla la búsqueda de un continuo crecimiento económico con consecuencias ambientales (Andersen 2007).

Por consiguiente, como lo indica Espaliat (2017, 20), la economía circular por principio es restaurativa y regenerativa, buscando lograr que los productos, componentes y materias primas mantengan su utilidad y valor máximo en todo momento.

Esto implica "desarrollar nuevos modelos económicos, considerando los diferentes campos de acción (el abastecimiento sostenible, el ecodiseño, la simbiosis industrial, la economía de la funcionalidad, el consumo responsable, la extensión de la vida útil, la gestión eficaz de los materiales o productos al final de su vida útil) que serán utilizados y articulados según las organizaciones para inclinarse hacia una economía circular" (INEN 2020, iv).

\section{La economía circular como una estrategia de desarrollo sostenible}

Frente a un mayor consumo global de recursos naturales y desafíos ambientales y socioeconómicos relacionados, la transición hacia una economía circular es de crucial importancia, especialmente desde que la Comisión Europea publicó su Plan de Acción de Economía Circular en diciembre de 2015 (Wilts 2017), por ello este tema ocupa un lugar destacado en la agenda política y estratégica en los países de la Comunidad Europea, China y Japón. 
Como señala Latouche (2007), el desarrollo sostenible ha sido la respuesta menos radical al modelo lineal, siendo la más acogida y la menos criticada, por cuanto permite el crecimiento económico, pero de una manera más armónica con la naturaleza y respetando las necesidades de las generaciones futuras, postulados compartidos por la economía circular.

Si bien el desarrollo sostenible se refiere a la integración de las metas de una calidad de vida elevada, la salud y la prosperidad con justicia social y al mantenimiento de la capacidad de la tierra para conservar la vida en toda su diversidad (INEN 2020), estas metas sociales, económicas y ambientales son interdependientes y se refuerzan mutuamente. El desarrollo sostenible puede considerarse como una vía para expresar las más amplias expectativas de la sociedad en su conjunto (INEN 2020).

Dentro de los Objetivos de Desarrollo Sostenible (ODS), la aplicación de una Economía Circular contribuirá a la consecución del Objetivo 12, que persigue garantizar modalidades sostenibles de consumo y producción, que enlaza con los Objetivos 8 "Trabajo Decente y Crecimiento Económico", 9 "Industria, Innovación e Infraestructura”, o el 11 "Ciudades y Comunidades Sostenibles", para, de manera conjunta, aumentar la competitividad y reducir la pobreza, y fomentar entre otras medidas, el uso eficiente de la energía y de los recursos ambientales, los empleos ecológicos y una mejor calidad de vida. (ES MAPAMA y MIMEINCO 2018, 15)

Varela (2018) señala que la economía circular al ser un modelo de producción y consumo competitivo y sostenible y, por ende, antagónico al obsoleto modelo lineal, persigue optimizar el uso de recursos, materias primas y energía, incorporando primero a la cadena de valor, y superando después las externalidades negativas.

La competitividad del modelo se basa en un diseño estratégico sostenible, capaz de ahorrar recursos y energía, generando productos menos contaminantes, posicionándose en una nueva dimensión de un mercado más consciente y comprometido con el planeta. Mientras que su sostenibilidad se deriva de la optimización en el uso de recursos, materiales y energías, lo que conlleva a la reducción de desperdicios y aprovechamiento de residuos. (Varela 2018, 26)

Por lo expuesto anteriormente, este investigador indica que la sostenibilidad del modelo radica en que la finitud de los recursos disponibles en el planeta no es esencial para que el sistema se mantenga o quiebre, pues tras 
el consumo del bien no se sucede el desecho, sino que se produce la fase de reciclado y/o reutilización (Varela 2018).

Por ello, la transformación a la economía circular está asociada con altas expectativas con respecto a los beneficios ecológicos y económicos. Como señala Wilts $(2017,1-2)$, los estudios enfatizan en los beneficios en cuatro niveles: utilización de recursos, medioambiente, economía y beneficios sociales, incluida la creación de nuevos empleos, así:

- Beneficios de disponibilidad de recursos al mejorar la seguridad de los recursos y reducir la dependencia de las importaciones.

- Beneficios ecológicos al generar menos impactos ambientales.

- Beneficios económicos, al gestar nuevas oportunidades para el crecimiento económico y la innovación.

- Beneficios sociales, al promover un comportamiento sostenible del consumidor y mayores posibilidades de empleo.

Es decir, en un sistema perfecto de economía circular el valor de los productos y materiales se mantiene durante el mayor tiempo posible, los residuos se reducen al mínimo, y los recursos se conservan dentro de la economía, aun cuando un producto haya llegado al final de su vida útil, con el fin de volverlo a utilizar repetidamente y seguir creando valor (García 2016).

\section{Metodología}

A través de un método exploratorio descriptivo, se identifican las publicaciones sobre economía circular, disponibles en bases de datos multidisciplinarias (como Proquest y Google Académico), repositorios (Cobuec), recursos de libre acceso a través de internet (Dianelt, Latindex, Redalyc, SciELO) y páginas web de organismos internacionales y nacionales (ISO, BSI, INEN). Se analizan alrededor de 50 documentos obtenidos aplicando criterios de búsqueda en español e inglés usando como palabra clave "economía circular" / "circular economy", y se selecciona la bibliografía utilizada. 
En síntesis, se llevó a cabo una investigación cualitativa documental, analizando fuentes secundarias respecto a la literatura generada sobre el tema. Una buena parte de los documentos revisados hacen referencia a la Ellen MacArthur Foundation, en su mayoría son artículos de origen europeo, y unos cuantos de otros países y de Latinoamérica. También se revisaron documentos institucionales y normas nacionales e internacionales.

Para la comprensión del tema investigado se aplicó un análisis de contenido cualitativo, buscando captar y comprender el significado de la economía circular, su aporte al desarrollo sostenible, su avance y consecuencias. Para ello se identificaron, categorizaron y enlazaron palabras y frases más utilizadas en la literatura internacional y nacional, analizando el texto y el contexto de exploración (Andreu-Abela 2002). Para, a través de un muestreo intencional/opinático (Palencia-Lefler 2008), realizar una descripción objetiva y sistemática, lo que permitió emitir conclusiones pertinentes.

\section{Discusión de resultados}

\section{La implantación de la economía circular}

Bajo la premisa de que una economía circular busca restaurar el capital financiero, manufacturero, humano, social y natural, con el fin de garantizar mejoras en los bienes y servicios, Ellen MacArthur Foundation propone un diagrama sistémico que presenta el flujo continuo de materiales técnicos y biológicos, denominado el "círculo de valor" (Ellen MacArthur Foundation 2020, párr. 2), que se describe en la figura 1.

Basicamente, dentro del círculo de valor se identifican seis acciones que pueden adoptar las organizaciones y los gobiernos para la transición a la economía circular. Descrito como marco RESOLVE: Regenerate (regenerar), Share (compartir), Optimise (optimizar), Loop (establecer bucles), Virtualise (virtualizar) y Exchange (intercambiar) (Varela 2018). 


\section{Figura 1}

\section{Diagrama sistémico de la economía circular}

Renovables

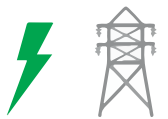

8 Recursos finitos

Regenerar Sustituir materiales

Desmaterializar Restauración

Agricultura/ cosecha/ recolección
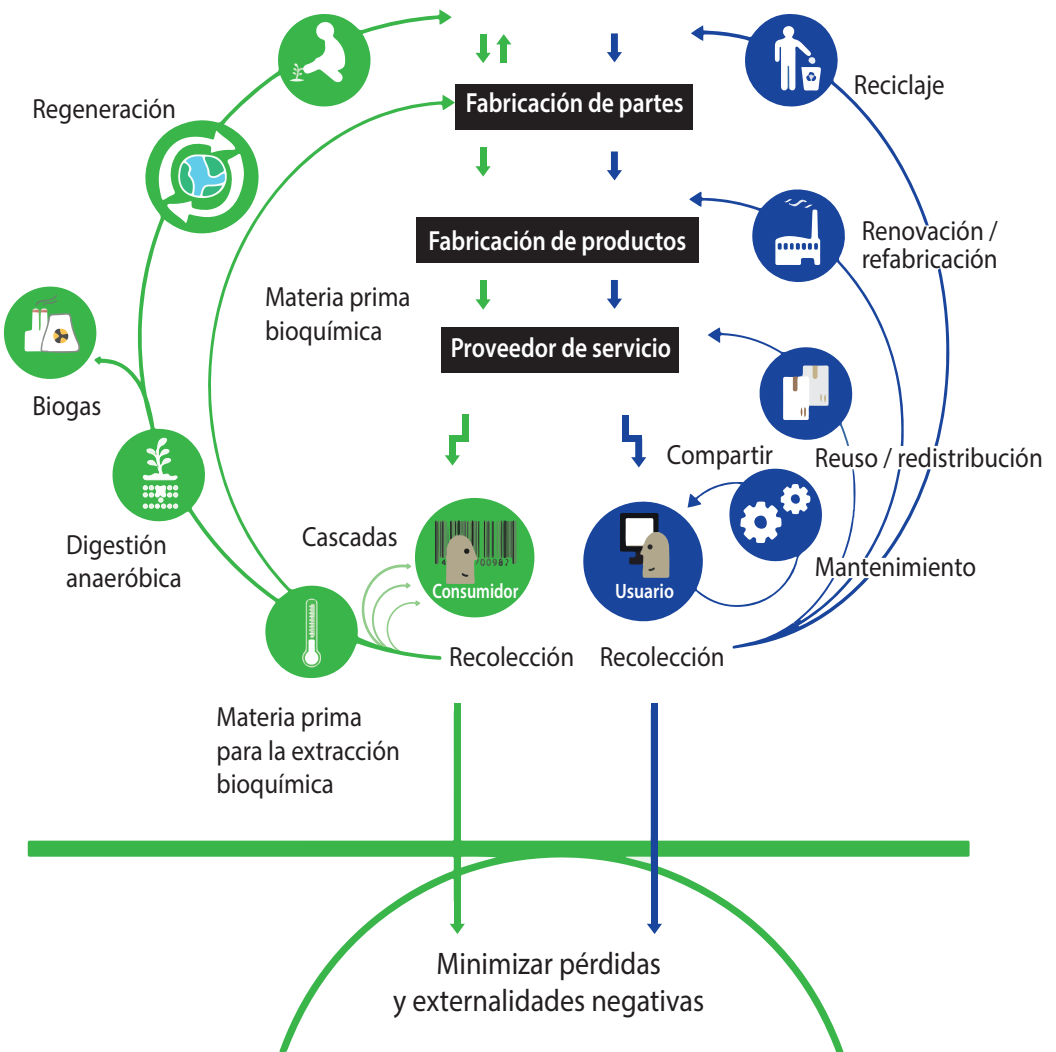

Fuente: Ellen MacArthur Foundation (2020). 
Además, la transición hacia una economía circular supone un cambio sistémico, en el que la investigación y la innovación, tanto tecnológica como no tecnológica (organizativas y sociales) desempeñan un papel clave para impulsar el cambio necesario en los modelos de producción y consumo de productos y servicios innovadores, que permitan a través de su adopción contribuir a la competitividad de las organizaciones (ES MAPAMA y MIMEINCO 2018).

\section{Transición hacia una economía circular}

Como se ha venido señalando, para la transición hacia una economía circular se requiere realizar cambios a lo largo de toda la cadena de valor del producto, desde su diseño hasta la logística inversa. Esto implica introducir un modelo sistémico de innovación transversal, tanto en el producto como en los procesos de producción (tecnología), así como también en la estructura de la organización, en los sistemas de financiación, en la cooperación entre empresas, en las políticas estratégicas y en la sociedad (Varela 2018).

Por ello, las organizaciones deberán mantener el valor de los productos y las materias primas el mayor tiempo posible, con un enfoque particular en actividades como la preparación para la reutilización, reparación y, en general, la vida útil de los productos, además de las actividades de diseño de productos duraderos, reparables y reciclables (Wilts 2017).

Sin embargo, del potencial de ganar-ganar de la economía circular, para investigadores como Korhonen, Honkasalo y Seppälä (2018), tiene sus limitaciones, mismas que deben resolverse para que pueda contribuir a la sostenibilidad global, señalando:

- Límites termodinámicos (los sistemas cíclicos que consumen recursos y generan desechos y emisiones).

- Límites del sistema (los problemas se desplazan a lo largo del ciclo de vida del producto).

- Límites planteados por la escala física de la economía (el efecto boomerang de la eficiencia y el consumo responsable puede enfrentar la paradoja de que la cultura de consumo no cambie).

- Límites planteados por la dependencia de ruta y bloqueo (dificultad de diferentes sociedades de cambiar hábitos y tecnología). 
- Límites de gobierno y gestión, estrategias interorganizacionales versus intraorganizacionales (para enfrentar nuevos desafíos de gestión de conflictos entre actores; además, los acuerdos internacionales actuales carecen de capacidad de coerción e impacto para asegurar su cumplimiento).

- Límites de definiciones sociales y culturales (definiciones de flujos físicos y de residuos aún no acordadas). (Korhonen, Honkasalo y Seppälä 2018, 41).

En medio de las ventajas y limitaciones expuestas, actualmente la economía circular tiene una gran fuerza inspiradora y constituye una razonable estrategia para lograr la tan anhelada sostenibilidad global. Por ello se requiere de un trabajo conjunto de gobierno, empresas, academia y sociedad en general.

\section{El camino recorrido hacia la economía circular, a nivel internacional y en Ecuador}

La transición de una economía lineal a una economía circular requiere de una actividad coordinada entre la administración pública, los sectores económicos, y el conjunto de la sociedad (ES MAPAMA y MIMEINCO 2018).

Si bien la Unión Europea (UE) ha venido utilizando todos los instrumentos de que dispone para transformar su economía, abriendo camino a nuevas oportunidades de negocio e impulsando la competitividad y la creación de empleo, las amplias medidas encaminadas a modificar todo el ciclo de vida del producto, sin limitarse a abordar la etapa del fin de vida, subrayan la clara voluntad de la Comisión Europea de transformar la economía de los países miembros y generar crecimiento sostenible (Comisión Europea 2015).

Como se señala en el Diario Oficial de la Unión Europea (2020), el concepto de economía circular se ha venido integrando entre sus países miembros, la existencia de un plan de acción a escala de la UE ha fomentado el desarrollo de planes nacionales y en determinadas regiones existen importantes conjuntos de estrategias, que han permitido superar ya las etapas del reciclado y la gestión de residuos. Actualmente vienen trabajando en hojas de ruta y en la creación de sinergias, buscando complementar con investigación y desarrollo, un entorno normativo adecuado, la educación de todas las 
partes interesadas y la información sobre el acceso al apoyo financiero para la transición hacia la circularidad.

En este contexto, países como Reino Unido y Francia, por ejemplo, a través de sus organismos de normalización, British Standards Institution (BSI) y la Asociación Francesa de Normalización (AFNOR), respectivamente, han desarrollado normas sobre economía circular. Así, BS 8000 es una norma británica, publicada en 2017, como una guía centrada en el concepto y principios de la economía circular, así como en la gestión de recursos (Pauliuk 2018), mientras que norma francesa AFNOR XP X30-901, publicada en 2018, aborda los requisitos y directrices de los sistemas de gestión de proyectos de economía circular.

A nivel mundial, algunas de las normas de la Organización Internacional de Estandarización (ISO) han venido siendo herramientas importantes y precursoras para promover una economía circular, entre ellas se pueden señalar, por ejemplo, a ISO 14001 (Sistema de gestión ambiental), ISO 26000 (Guía de responsabilidad social).

Al interior de ISO se ha creado el comité ISO/TC 323, en el cual se están desarrollando normas sobre economía circular, que proporcionarán requisitos, guías y métodos para medir aspectos como la durabilidad o la capacidad de actualización de un producto, la facilidad con que se puede reparar o reciclar y garantizar la calidad de los materiales reciclados. Además, los documentos normativos se basarán en siete principios:

- Obtención sostenible de recursos.

- Ecodiseño.

- Simbiosis industrial.

- Economía de funcionalidad.

- Consumo responsable.

- Extensión del ciclo de vida del producto.

- Gestión eficiente del fin de la vida útil de productos y materiales.

La ISO / TC 323 tiene como objetivo cubrir todos los aspectos de una economía circular, incluida la contratación pública, la producción y distribución, el final de la vida útil y áreas más amplias, como el cambio de comportamiento en la sociedad y la evaluación, como algún tipo de huella de circularidad o índice (ISO 2020). 
También se beneficiará de la vinculación con los muchos otros comités técnicos de ISO dedicados a estándares relacionados, como los de compras sostenibles, calidad y gestión ambiental, responsabilidad social y más (ISO 2020). En la figura 2 se describe la relación ISO y los objetivos de la economía circular.

Figura 2

ISO y los objetivos de la economía circular bajo las tres dimensiones del desarrollo sostenible: ambiental, económico y social

\section{Comité ISOTC 323}

Comité internacional ISO TC 323

Economía circular

- La organización de normalización internacional ISO ha creado el comité ISO/TC 323 en el cual se desarrollarán normas sobre economía circular.

Los documentos normativos

se basarán en siete principios:

- Obtención sostenible de recursos

- Ecodiseño

- Simbiosis industrial

- Economía de funcionalidad

- Consumo responsable

- Extensión de ciclos de vida del producto

- Gestión eficiente del fin de la vida útil

de productos y materiales.

\section{Economía circular y los siete principios}

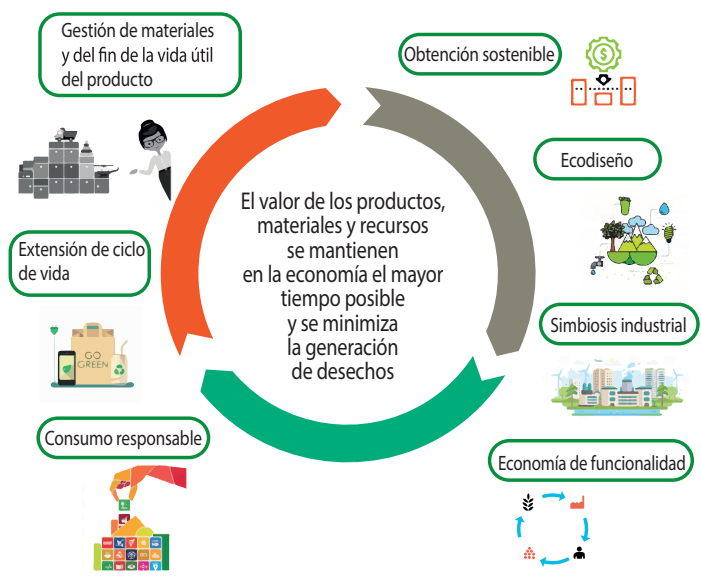

Fuente: Díaz y Santana (2019).

Así mismo, el trabajo de ISO / TC 323 busca contribuir directamente a muchos Objetivos de Desarrollo Sostenible (ODS) de las Naciones Unidas, como el ODS 8, Trabajo decente y crecimiento económico; ODS 12, Consumo y producción responsable; ODS 13, Acción climática; y ODS 15, La vida en la tierra (ISO 2020).

Por lo tanto, este comité tiene la intención de producir un conjunto de principios acordados internacionalmente, terminología, un marco de lo que 
es una economía circular y desarrollar un estándar de sistema de gestión. También trabajará en modelos de negocio alternativos y métodos para medir y evaluar la circularidad, pues existe una urgencia reconocida para avanzar hacia una economía circular, debido a los efectos del agotamiento de los recursos y la biodiversidad, el cambio climático y las crecientes desigualdades entre los países relacionadas con los patrones de producción y consumo del mundo (ISO 2020).

"Esto es particularmente cierto en los países en desarrollo que han tendido a soportar la peor parte de las desigualdades de riqueza y desperdicio en el mundo desarrollado" (ISO 2020, párr. 10). Por ello, se esperaría que las economías emergentes se beneficien de la circularidad y dejen de seguir tendencias tecnológicas basadas en modelos económicos obsoletos en contra de sus necesidades reales.

En Ecuador, el Ministerio de Producción, Comercio Exterior, Inversiones y Pesca ha venido realizando un trabajo conjunto con el Servicio de Normalización Ecuatoriano (INEN) para implementar una marca de economía circular en las industrias que cumplan con requisitos establecidos en una norma técnica adoptada por este organismo, basada en la normativa de economía circular elaborada por la Organización Francesa de Normalización (AFNOR).

A fin de que las organizaciones ecuatorianas empiecen a involucrarse con este tema, se han iniciado talleres de capacitación, y aún está en proceso la elaboración del Libro Blanco de la Economía Circular que se preveía estaría listo a finales de 2019, y que reunirá estudios que permitan definir una línea base sobre el estado del país en torno a este modelo y al futuro para Ecuador.

Es importante señalar que también se encuentra en discusión, en la Asamblea Nacional, la Ley Orgánica de Economía Circular, cuyo texto ya ha sido aprobado para debate con los diferentes grupos de interés.

Entre los motivos que han llevado a la generación de este proyecto de ley está la necesidad e importancia de que el país entre en un proceso de transición de la economía lineal a la economía circular, buscando generar nuevas ventajas competitivas y sostenibles que generen beneficios económicos y ambientales para la sociedad ecuatoriana (EC 2020, 2; documento de trabajo), por lo que resulta mandatorio: 
Incorporar en el ordenamiento jurídico ecuatoriano principios, obligaciones, reglas etc., con el objetivo principal de conseguir el máximo valor y uso de todos los recursos, productos y residuos, fomentando la gestión racional y sostenible de los recursos, promoviendo la reducción de la generación de residuos, su reciclaje, reutilización y valorización, el ahorro energético y reduciendo las emisiones de gases contaminantes y de efecto invernadero.

Y así mismo, ayudar a las empresas y consumidores a entrar en esta transición, abarcando todo el ciclo, desde la producción hasta el consumo, gestión de residuos, reciclaje y reutilización al final del proceso (EC 2020).

Otro de los objetivos es buscar una transición que permita a las organizaciones alcanzar los requisitos establecidos en la norma francesa AFNOR XP X30-901:2018, actualmente ya adoptada como norma ecuatoriana NTE INEN-AFNOR XP X30-901:2020 (EC 2020, art. 2), y motive la gestión de las empresas ecuatorianas con normativas que aporten al sistema de circularidad en diferentes procesos, como son: el ciclo de vida de un producto, etiquetado ambiental, sistema de gestión ambiental, huella de agua y huella de carbono en productos.

\section{Conclusiones}

De acuerdo con la revisión bibliografía, se puede decir que la economía circular está siendo acogida, por numerosos actores en los ámbitos empresarial, académico y gubernamental, como una estrategia de desarrollo sostenible, que promueve una mayor protección ambiental y beneficio social, pero también como una estrategia competitiva en el mundo empresarial.

La economía circular como un modelo que pretende cambiar la concepción de la producción, ha venido implementándose como una enmienda a muchos de los problemas generados por un sistema de producción y consumo lineal. Para ello esta debe garantizar la rentabilidad y viabilidad económica del cambio de paradigma, así como la sensibilización y otra mentalidad de todas las partes interesadas, motivando su concienciación medioambiental en la búsqueda del bienestar y salud de los consumidores y sus familias.

Es indispensable el desarrollo de un enfoque sistémico para el diseño de procesos (como un elemento clave desde sus etapas iniciales), productos/ 
servicios y modelos de negocio innovadores que creen un valor agregado económico a partir de recursos naturales limitados. Esto puede ayudar a las organizaciones a volverse más resistente a las conmociones externas y mejorar su competitividad global.

La economía circular, además de una mayor seguridad en la cadena de suministro, presenta para las empresas numerosas oportunidades, como el surgimiento de nuevas líneas de negocio y servicios, así como una reducción de insumos y materias primas, un reaprovechamiento de los residuos producidos y menores costes de gestión de estos (ES MAPAMA y MIMEINCO 2018).

Este modelo económico conlleva un cambio de paradigma a todo nivel, empezando por las políticas públicas, que deben considerar tanto el carácter transversal como el carácter gradual del concepto jurídico de economía circular; por los ciudadanos, que deben tomar conciencia de sus necesidades reales de consumo; por las empresas, que deben buscar ser sostenibles a través de resultados económicos, sociales y ambientales; y por la academia, que debe aportar investigaciones y conocimiento.

Se esperaría que los países en desarrollo se beneficien de la circularidad, ya que este nuevo modelo económico estimula ideas creativas e innovadoras para un mejor uso de los recursos y un crecimiento sostenible.

Además, el tema de circularidad también va a ser parte de las mesas de negociación de los diferentes tratados comerciales y habrá influencia en el comercio mundial, habiendo apertura o cierre de mercados de acuerdo con las estrategias que defina cada país.

Sin embargo del camino recorrido hacia la economía circular por parte de algunos países como los de la Unión Europea, tanto en estos como en países en vías de desarrollo, resulta fundamental superar barreras políticas, barreras relacionadas con la percepción pública o con la gobernanza de las infraestructuras y barreras financieras, aún presentes hasta la fecha.

La evolución de la economía mundial y la proyección histórica de la industria han llevado a tomar conciencia de la importancia de un cambio hacia una economía circular, la cual ha llegado a influir en los postulados de la Industria 4.0 y la "cuarta revolución industrial".

Una limitación de este estudio es la escasa evidencia empírica local sobre el tema, aunque hay esfuerzos que se vienen haciendo desde la Asamblea 
Nacional con la Ley de Economía Circular, así como también el Ministerio del Ambiente, conjuntamente con el Ministerio de Producción, que están desarrollando el Libro Blanco de la Economía Circular, la norma INEN vigente sobre economía circular -sistemas de gestión de proyectos de economía circular-, requisitos y directrices (AFNOR XP X30-901:2018, IDT) (INEN 2020); sin embargo, aún no existe una estrategia clara, así como tampoco políticas públicas que guíen a las organizaciones ecuatorianas a reconsiderar, de manera holística, la gestión de los recursos a través de todo el ciclo de vida de un producto/servicio y sortear las dificultades a las que se podrían enfrentar en el marco de una economía lineal imperante. Este artículo pretende ser un aporte teórico-académico del avance de la economía circular en el Ecuador.

\section{Recomendaciones}

Ante la realidad actual de la pandemia mundial ocasionada por el COVID-19, las economías de los países deberán ser restauradas, especialmente la de los países pobres, por lo que la economía circular podría ser un medio práctico para alcanzar los grandes objetivos sociales, económicos y ambientales, como los ODS y aquellos vinculados con los tratados internacionales contra el cambio climático.

Por ello, resulta esencial inscribir a nivel de política pública técnicas y estrategias fundamentadas en la circularidad a lo largo de toda la cadena de valor de los productos y servicios, y de todo el ciclo técnico y biológico de los materiales. Se deben incorporar buenas prácticas intrínsecas a la concepción de la economía circular, como reducir, reciclar, rehabilitar, reparar, redistribuir, restaurar, rediseñar, repensar, refabricar, reutilizar y recuperar. Como lo señala en su investigación Varela (2018), de esta manera se logrará la transición a un modelo circular que asegure la continuidad y la regeneración de los materiales y productos y, con ello, de la vida en todas sus dimensiones, así como restituir el daño causado por el modelo lineal.

Desde el Estado, se deberían generar incentivos a las formas innovadoras y más eficientes de producir y consumir; así como también establecer prácticas de gobernanza basadas en la normativa adecuada que garantice niveles 
de calidad, productividad e innovación, que impulsen la competitividad de las organizaciones, lo que conlleve a la creación de empleo y a un crecimiento sostenible.

Además, siguiendo el ejemplo de otros países como España, fomentar la colaboración y la coordinación entre los agentes económicos, sociales, academia y las administraciones públicas para hacer frente, de forma común, a los retos medioambientales, económicos, sociales y tecnológicos.

\section{Referencias}

Andersen, Mikael Skou. 2017. "An Introductory Note on the Environmental Economics of the Circular Economy”. Sustainability Science 2 (1): 133-140. 10.1007/s11625-006-0013-6.

Andreu-Abela, Jaime. 2002. "Las técnicas de análisis de contenido: una revisión actualizada". Fundación Centro Estudios Andaluces 20 (3): 1-34. https://bit.ly/30wioY6.

Asociación Francesa de Normalización (AFNOR). 2018. AFNOR XP X30-901 (Economía circular - Sistemas de gestión de proyectos de economía circular - Requisitos y directrices. París: AFNOR.

Chaves, Rafael, y José Luis Monzón. 2018. “La economía social ante los paradigmas económicos emergentes: innovación social, economía colaborativa, economía circular, responsabilidad social empresarial, economía del bien común, empresa social y economía solidaria”. CIRIEC-España, Revista de Economía Pública, Social y Cooperativa (93): 5-50. 10.7203/CIRIEC-E.93.12901.

Comisión Europea. 2015. "Cerrar el círculo: la Comisión adopta un ambicioso paquete de nuevas medidas sobre la economía circular para impulsar la competitividad, crear empleo y generar crecimiento sostenible". Bruselas: Press releases database. Comunicado de Prensa. Accedido 2 diciembre de 2019. https://bit.ly/2yoKEQH.

Contreras Castañeda, Eduin, Rafael Tordecilla Madera y Julián Silva Rodríguez. 2013. "Revisión de estudios de caso de carácter cualitativo y exploratorio en logística inversa". Revista EIA 10 (20): 153-164. http:/dx.doi.org/10.14508/reia.2013.10.20.153-165.

Diario Oficial de la Unión Europea. 2020. Dictamen del Comité Económico y Social Europeo sobre "Desarrollar sinergias entre las distintas hojas de ruta de la economía circular". Accedido 29 de marzo. https://bit.ly/2BdbKvb.

Díaz, César, y Andrea Santana. 2019. “Economía circular”. Ponencia presentada en el Comité ISO TC 323 del INEN para la adopción de la norma AFNOR XP X30-901:2018, Quito, 15 de agosto.

EC. 2020. Resolución MPCEIP-SC-2020-0028-R. Registro Oficial 345, Edición Especial, 13 de febrero.

---. 2020. Proyecto de Ley Orgánica de Economía Circular. Documento de trabajo, 20 de abril. 
Ellen MacArthur Foundation. 2015. Growth Within: A Circular Economy Vision for a Competitive Europe. Cowes: Ellen MacArthur Foundation. https://bit.ly/3biDdJc.

---. 2019. Completing the Picture: How the Circular Economy Tackles Climate Change. Cowes: Ellen MacArthur Foundation. https://bit.ly/3eB7DIM.

--- 2020. "Circular de economía". Ellen MacArthur Foundation. Accedido 31 de marzo. https: //bit.ly/3cvVaUL.

ES Ministerio de Agricultura y Pesca, Alimentación y Medio Ambiente (MAPAMA) y Ministerio de Economía, Industria y Competitividad (MIMEINCO). 2018. España circular 2030: estrategia española de economía circular. Madrid: Ministerio de Economía, Industria y Competitividad.

Espaliat, Mauricio. 2017. Economía circular y sostenibilidad: nuevos enfoques para la creacion de valor. Santiago de Chile: CreateSpace. https://bit.ly/2Vol0oe.

Fundación COTEC. 2017. "Informe COTEC". Fundación COTEC. https://bit.ly/2Vmxmx9.

García García, Sara. 2016. "Economía circular: la Unión Europea impulsa reformas sobre la base de un tema crucial, la gestión de residuos, con el fin de alcanzar mejoras económicas y medioambientales". Actualidad Jurídica Ambiental 57: 1-11.

Ghisellini, Patrizia, Catia Cialani y Sergio Ulgiati. 2015. "A Review on Circular Economy: the Expected Transition to a Balanced Interplay of Environmental and Economic Systems. Journal of Cleaner Production 114: 1-22. http://dx.doi.org/10.1016/j.jclepro.2015.09.007.

Guohui, Song, y Li Yunfeng. 2012. "The Effect of Reinforcing the Concept of Circular Economy in West China Environmental Protection and Economic Development. 2011 International Conference on Environmental Science and Engineering (ICESE 2011)". Procedia Environmental Sciences 12: 785-792. 10.1016/j.proenv.2012.01.349.

INEN (Servicio Ecuatoriano de Normalización). 2020. NTE INEN-AFNOR XP X30-901 (Economía circular - Sistemas de gestión de proyectos de economía circular - Requisitos y directrices, AFNOR XP X30-901:2018, IDT). Quito: INEN.

ISO (Organización Internacional de Estandarización). 2019. "Conectando los puntos en una economía circular: se acaba de formar un nuevo comité técnico de ISO”. Accedido 27 de febrero de 2020. https://bit.ly/2XP1Lpt.

Korhonen, Jouni, Antero Honkasalo y Jyri Seppälä. 2018. "Circular Economy: The Concept and its Limitations". Ecological Economics 143: 37-46. https://doi.org/10.1016/j.ecolecon.2017.06.041.

Lett, Lina A. 2014. "Las amenazas globales, el reciclaje de residuos y el concepto de economía circular". Revista Argentina de Microbiología 46 (1): 1-2. https://bit.ly/3bnBe6n.

Martínez, Federico, y Jesús Londoño. 2012. "El pensamiento sistémico como herramienta metodológica para la resolución de problemas". Revista Soluciones de Posgrado EIA 8: 43-65.

Palencia-Lefler, Manuel. 2008. "La incomunicación interna en la Universidad española". Revista Latina de Comunicación Social (63): 1-20. https://bit.ly/2Ca0mku. 
Pauliuk, Stefan. 2018. "Critical Appraisal of the Circular Economy Standard BS 8001:2017 and a Dashboard of Quantitative System Indicators for its Implementation in Organizations". Resources, Conservation \& Recycling 129: 81-92. https://doi.org/10.1016/j.resconrec.20 17.10.019.

Priede Bergamini, Tiziana, e Ivan Hilliard. 2019. "La economía circular en la industria alimentaria". Economistas sin fronteras. Responsabilidad social corporativa en la industria alimentaria. Dossieres EsF (35): 36-40.

Prieto-Sandoval, Vanessa, Carmen Jaca y María Ormazabal. 2017. "Economía circular: relación con la evolución del concepto de sostenibilidad y estrategias para su implementación". Memoria Investigaciones en Ingeniería 15: 85-95. https://bit.ly/3frT75L.

Santamaría Arinas, René Javier. 2019. "Economía circular: líneas maestras de un concepto jurídico en construcción”. Revista Catalana de Dret Ambiental 10 (1): 1-37. https://doi. org $/ 10.17345 /$ rcda2569.

Varela Menéndez, Jacobo. 2018. "La economía circular: una propuesta de futuro para España y Europa". Trabajo de grado, Universidad de Coruña, Coruña. https://ruc.udc.es/dspace/ handle/2183/21053.

Wilts, Henning. 2017. "Key Challenges for Transformations Towards a Circular Economy - The Status Quo in Germany". International Journal of Waste Resources 7 (262): 1-5. $10.4172 / 2252-5211.1000262$.

World Economic Forum. 2014. "Towards the Circular Economy: Accelerating the Scale-up Across Global Supply Chains". Accedido 15 de marzo de 2020. https://bit.ly/2XQmUj8. 